\title{
Determination of Talniflumate and Niflumic Acid in Human Plasma by Liquid Chromatography-Tandem Mass Spectrometry
}

\author{
Wonku KANG ${ }^{\dagger}$ and Kibum KIM \\ College of Pharmacy, Catholic University of Daegu, Kyoungbuk 712-702, South Korea
}

\begin{abstract}
A simple and rapid quantification method was developed for determining both talniflumate and niflumic acid in human plasma. After simple protein precipitation with acetonitrile, the analytes were chromatographed on a reversed-phase $\mathrm{C}_{18}$ column and detected by LC/MS/MS with electrospray ionization. The assay accuracy and precision were within the FDA guidance for the analytical method validation. This method was used to measure the plasma concentrations of both compounds from healthy subjects after a single oral dose of talniflumate, $740 \mathrm{mg}$.
\end{abstract}

(Received October 7, 2008; Accepted February 12, 2009; Published April 10, 2009)

\section{Introduction}

Talniflumate is a potent analgesic and anti-inflammatory drug that is widely prescribed for rheumatoid diseases. ${ }^{1}$ It was synthesized by the esterification of a carboxyl group of niflumic acid with the phthalidyl moiety, and it exerts activity in the body through conversion to niflumic acid. ${ }^{2}$

In previous work, niflumic acid in human plasma has been mainly determined using high-performance liquid chromatography with ultraviolet detection, ${ }^{3-6}$ and gas chromatography with a mass spectrometry that provided more sensitivity. ${ }^{7}$ Although a method has recently appeared on line, they used a single mass spectrometer, and only dealt with niflumic acid. ${ }^{8}$ Therefore, we develop a simultaneous method for determining both talniflumate and niflumic acid in human plasma using LC/MS/MS after a simple protein precipitation with an organic solvent. This method was successfully applied to characterize the pharmacokinetics of niflumic acid in humans following an oral administration of talniflumate.

\section{Experimental}

\section{Reagents and materials}

Talniflumate, niflumic acid and ramipril (internal standard, IS) were kindly donated by Sam-Nam Pharmaceutical Ltd. (ChungNam, South Korea). HPLC-grade organic solvent was purchased from Merck Co. (Darmstadt, Germany), and all the other chemicals and solvents were of the HPLC grade available.

\section{Preparation of standards and quality controls}

Talniflumate, niflumic acid and IS were dissolved in acetonitrile at $1 \mathrm{mg} / \mathrm{ml}$, respectively. The standard solutions were serially diluted with acetonitrile and added to drug-free plasma to obtain concentrations of $10,20,50,100,500$ and $2000 \mathrm{ng} / \mathrm{ml}$ for talniflumate and niflumic acid. Calibration graphs in plasma were derived from the peak-area ratios of

$\dagger$ To whom correspondence should be addressed.

E-mail:wonkuk@cu.ac.kr talniflumate and niflumic acid to IS with a linear regression.

Quality controls were prepared daily in $90 \mu \mathrm{l}$ of blank human plasma by adding $10 \mu \mathrm{l}$ of a standard solution to evaluate the inter- and intra-day precision and accuracy of this assay method.

Characterization of the product ions using tandem mass spectrometry

Ten nanograms per milliliters of talniflumate, niflumic acid and IS solutions were infused into the mass spectrometer separately at a flow rate of $10 \mu \mathrm{l} / \mathrm{min}$ to characterize the precursor and product ions of each compound. The precursor ions, and the pattern of fragmentation for analytes and IS were monitored using negative and positive ion modes, respectively. The major peaks observed in the MS/MS scan were used to quantify each molecule.

\section{Analytical system}

The concentrations of analytes in human plasma were quantified using liquid chromatography-mass spectrometry with a PE SCIEX API $4000^{\mathrm{TM}}$ LC/MS/MS System (Applied Biosystems, Foster City, CA) equipped with an electrospray ionization interface used to generate both negative ions $[\mathrm{M}-\mathrm{H}]$ for niflumic acid and positive ions $[\mathrm{M}+\mathrm{H}]^{+}$for talniflumate and IS. The compounds were separated on a reversed-phase column $\left(\mathrm{XTerra}^{\circledR} \mathrm{C}_{18}, 50 \times 2.1 \mathrm{~mm}\right.$ internal diameter, $3.5 \mu \mathrm{m}$ particle size; Waters, USA) with a mobile phase consisting of acetonitrile/0.1\% formic $\operatorname{acid}_{(\mathrm{aq})}[75: 25(\mathrm{v} / \mathrm{v})]$. The mobile phase was eluted using an HP 1100 series pump (Agilent, Wilmington, DE, USA) at $0.3 \mathrm{ml} / \mathrm{min}$, and the pressure was $\sim 60 \mathrm{psi}$. The turboion spray interface was operated in the positive ion mode at $5500 \mathrm{~V}$ and $350^{\circ} \mathrm{C}$. The operating conditions were optimized by the flow injection of a mixture of all anlalytes, and were determined as follows: nebulizing gas flow, $1.04 \mathrm{~L} / \mathrm{min}$; auxiliary gas flow, $4.0 \mathrm{~L} / \mathrm{min}$; curtain gas flow, $1.44 \mathrm{~L} / \mathrm{min}$; orifice voltage, $80 \mathrm{~V}$; ring voltage, $400 \mathrm{~V}$; collision gas (nitrogen) pressure, $3.58 \times 10^{-5}$ torr. Quantitation was performed by multiple reaction monitoring (MRM) of the precursor ions and the related product ions for talniflumate and niflumic acid using the internal standard method with the peak-area ratios. The mass transition used for talniflumate, niflumic acid and IS were $\mathrm{m} / \mathrm{z} 415.1 \rightarrow 132.9$ (declustering potential, $76 \mathrm{eV}$; collision 
energy, $33 \mathrm{eV}$; dwell time, $200 \mathrm{~ms}), \mathrm{m} / \mathrm{z}, 280.9 \rightarrow 236.5$ (declustering potential, $70 \mathrm{eV}$; collision energy, $24 \mathrm{eV}$; dwell time, $200 \mathrm{~ms}$ ) and $417.2 \rightarrow 234.1$ (declustering potential, $71 \mathrm{eV}$; collision energy, $31 \mathrm{eV}$; dwell time, $200 \mathrm{~ms}$ ), respectively. Quadrupoles Q1 and Q3 were set on unit resolution. The analytical data were processed by Analyst software (Ver. 1.4.1, Applied Biosystems).

\section{Sample preparation}

Nine-hundred microliters of internal standard $(50 \mathrm{ng} / \mathrm{ml})$ were added to $0.2 \mathrm{ml}$ of the plasma, followed by $10 \mathrm{~s}$ of vigorous vortex-mixing. The mixture was centrifuged at $13200 \mathrm{rpm}$ for 10 $\mathrm{min}$, and further diluted with acetonitrile by 3 -fold. One microliter of the solution was injected onto an analytical column. ${ }^{9,10}$

\section{Validation procedure}

The validation parameters were: selectivity, matrix effect, precision and accuracy. Five batches of blank heparinized human plasma were screened to determine the specificity. To study the matrix effect, blank plasma samples were processed and spiked later to obtain concentrations at 0.1 and $1 \mu \mathrm{g} / \mathrm{ml}$. The response (area) was compared with standard solutions directly injected at the same levels. The precision and accuracy of the intra- and inter-day assay validation were estimated using an inverse prediction of the concentration of the quality controls at $10,50,500,2000 \mathrm{ng} / \mathrm{ml}$ from the calibration curves.

For a stability study in plasma, control drug-free plasma samples were spiked with 50 and $500 \mathrm{ng} / \mathrm{ml}$ for analytes. Shortterm stability was assessed after $24 \mathrm{~h}$ of storage at room temperature; long-term stability was assessed after 3 weeks of storage in a freezer at $-70^{\circ} \mathrm{C}$. The stability of talniflumate and niflumic acid in plasma samples was tested after three freezethaw cycles $\left(-70^{\circ} \mathrm{C}\right.$ to room temperature). The stability in extracts was also examined after $24 \mathrm{~h}$ of storage at $4^{\circ} \mathrm{C}$.

\section{Clinical application}

Twenty-four healthy subjects, who gave written informed consent, took part in this study. Health problems, drug or alcohol abuse, and abnormalities in laboratory screening values were exclusion criteria. This study was approved by the Institutional Review Board of College of Medicine, Kyoungpook National University (Daegu, Korea). After an overnight fast, all of the subjects were given a single $740-\mathrm{mg}$ oral dose of talniflumate. Blood samples $(5 \mathrm{ml})$ were taken before and 0.5 , $1,1.5,2,2.5,3,4,5,6,8,10$ and $12 \mathrm{~h}$ after drug administration. Plasma was separated by centrifugation at $3000 \mathrm{rpm}$ for $10 \mathrm{~min}$, and kept frozen at $-70^{\circ} \mathrm{C}$ until analysis.

\section{Results and Discussion}

\section{Mass spectra}

Precursor ions for talniflumate, niflumic acid and IS, and their corresponding product ions were determined from spectra obtained during the infusion of standard solutions into a mass spectrometer using an eletrospray ionization source, which operated in a multiple reaction monitoring mode with collision nitrogen gas in Q2 of the MS/MS system. Whereas niflumic acid mainly produced deprotonated molecules at $\mathrm{m} / \mathrm{z}$. 280.9, talniflumate and IS generated protonated ones at $\mathrm{m} / \mathrm{z} 415.1$ and 417.2, respectively. Their proposed product ions were scanned in Q3 after collisions with nitrogen in Q2 at $\mathrm{m} / z, 132.9$ and 236.5 for talniflumate and niflumic acid, respectively, and at $\mathrm{m} / \mathrm{z} 234.1$ for IS, which are the most sensitive product ions for quantification (Fig. 1).
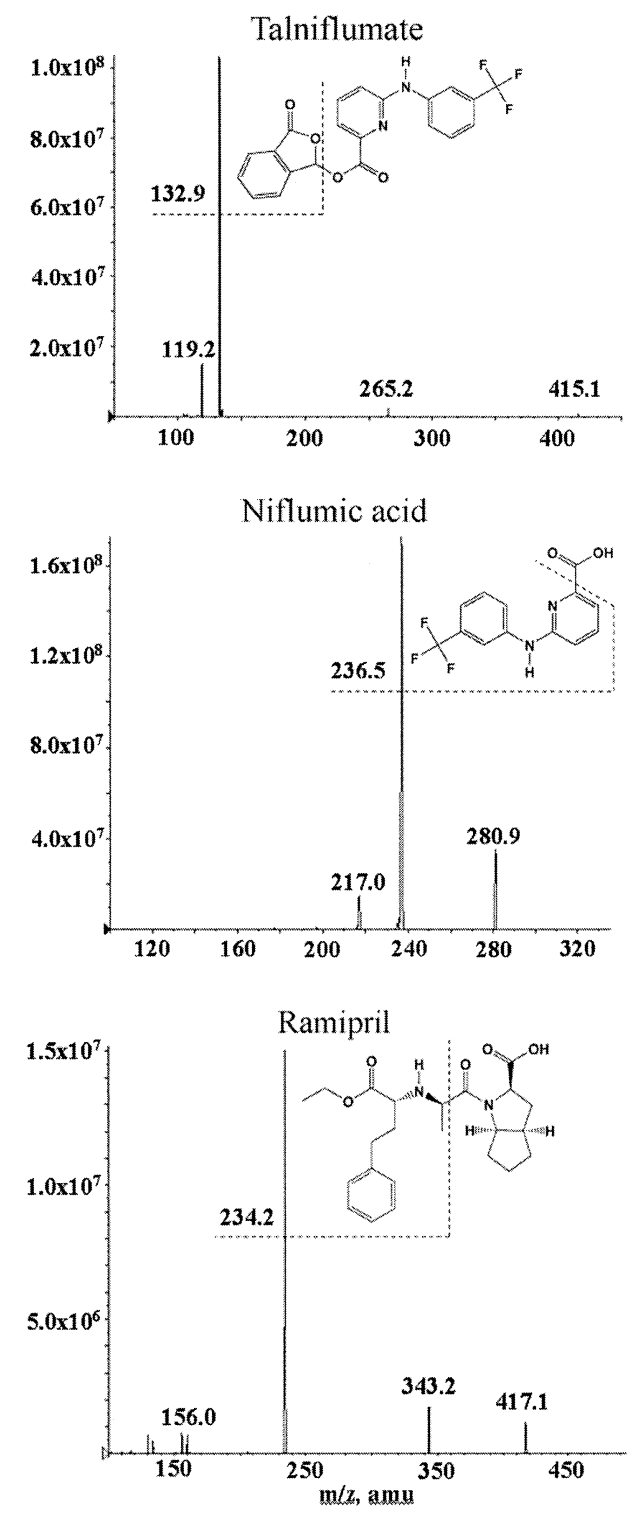

Fig. 1 Mass spectra of talniflumate, niflumic acid and ramipril obtained using the electrospray ionization mode.

\section{Determination of talniflumate and niflumic acid}

There were no interfering peaks at the elution times for either analytes (talniflumate, $1.1 \mathrm{~min}$; niflumic acid, $0.9 \mathrm{~min}$ ) or IS (ramipril, $0.5 \mathrm{~min}$ ), indicating that endogenous compounds did not hinder the measurement of talniflumate and niflumic acid in human plasma (Fig. 2, left column). The middle column represented typical chromatograms for the plasma spiked with 1 $\mathrm{ng} / \mathrm{ml}$ for talniflumate and $100 \mathrm{ng} / \mathrm{ml}$ for niflumic acid together with $50 \mathrm{ng} / \mathrm{ml}$ for IS. The plasma sample from a volunteer is shown in the right-hand column.

\section{Linearity, matrix effect, precision, accuracy and stability}

The calibration curves in plasma provided a reliable response from 10 to $2000 \mathrm{ng} / \mathrm{ml}$ for talniflumate as well as niflumic acid $\left(r^{2}>0.9999\right)$. The ion suppressions due to the matrix components were about 14 and $94 \%$ for talniflumate and niflumic acid, respectively.

The intra- and inter-day precision and accuracy of this method are listed in Table 1. The coefficients of variation of the precision of the intra- and inter-day validation were less than 

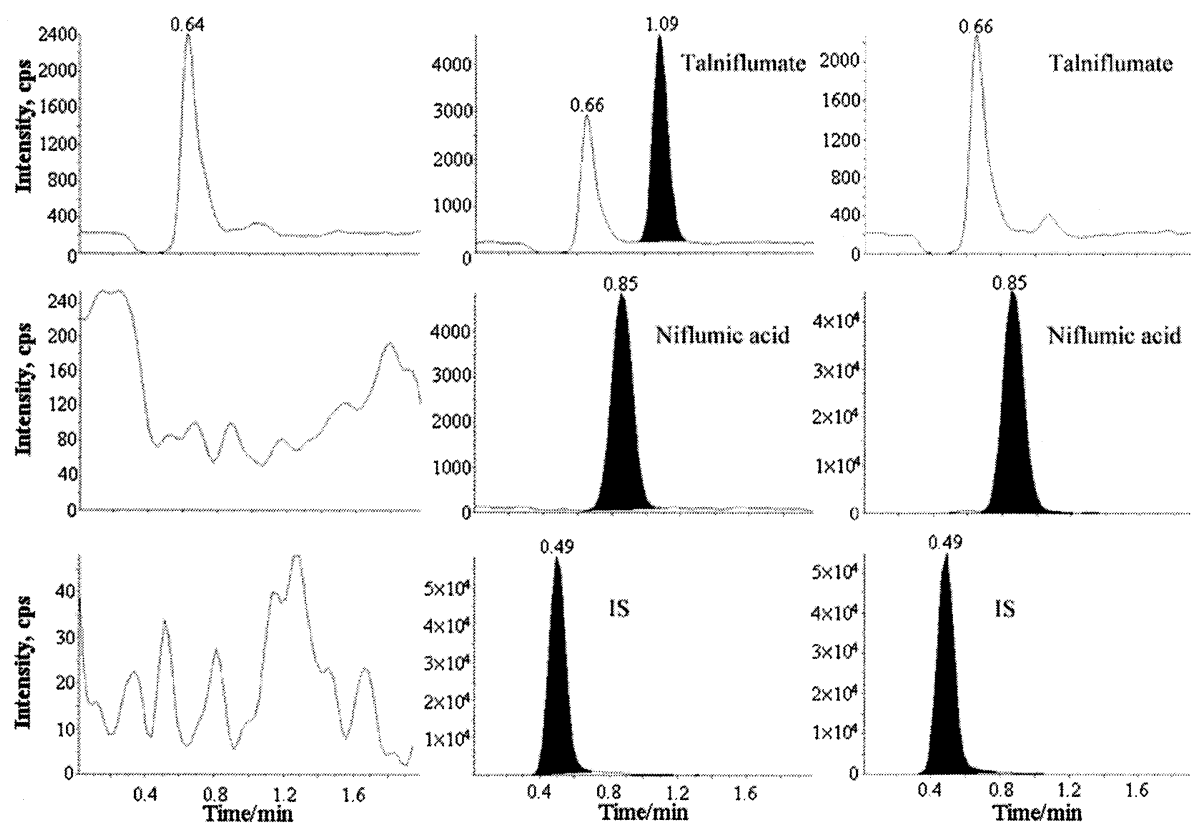

Fig. 2 Chromatograms of talniflumate, niflumic acid and ramipril. Left column, blank plasma; middle column, plasma spiked with $50 \mathrm{ng} / \mathrm{ml}$ of talniflumate and niflumic acid, respectively; right column, plasma sample equivalent to $890 \mathrm{ng} / \mathrm{ml}$ for niflumic acid from a volunteer $2 \mathrm{~h}$ after the oral dose of $740 \mathrm{mg}$ talniflumate.

Table 1 Precision and accuracy of the intra- and inter-day assay $(n=5)$

\begin{tabular}{|c|c|c|c|c|}
\hline \multirow{2}{*}{ Concentration/ng ml ${ }^{-1}$} & \multicolumn{2}{|c|}{ Intra-day } & \multicolumn{2}{|c|}{ Inter-day } \\
\hline & Talniflumate & Niflumic acid & Talniflumate & Niflumic acid \\
\hline 10 & $106.5 \pm 7.3^{\mathrm{a}}(6.9)^{\mathrm{b}}$ & $117.8 \pm 2.7$ & $108.6 \pm 9.5(8.7)$ & $98.2 \pm 13.3(13.5)$ \\
\hline 100 & $102.1 \pm 5.6$ & $101.8 \pm 7.5$ & $106.5 \pm 7.8(7.3)$ & $102.2 \pm 6.3$ \\
\hline 500 & $98.8 \pm 8.5(8.6)$ & $105.4 \pm 11.6(11.0)$ & $103.9 \pm 8.3(8.0)$ & $97.3 \pm 5.5 \quad(5.7)$ \\
\hline 2000 & $95.5 \pm 6.7(7.0)$ & $95.5 \pm 11.2(11.7)$ & $99.8 \pm 6.4(6.4)$ & $96.2 \pm 5.0$ \\
\hline
\end{tabular}

a. Accuracy (mean $\% \pm$ S.D.). b. CV, coefficient of variance (\%).

11.7 and $13.5 \%$, respectively. The accuracy of the method ranged from 96 to $118 \%$. Talniflumate and niflumic acid were stable in plasma at room temperature for up to at least $24 \mathrm{~h}$; they also remained intact at $-70^{\circ} \mathrm{C}$ for up to 3 weeks. With respect to the runtime stability of the processed samples, no significant loss of the two compounds was observed at room temperature, and no degradation was observed after three cycles of freezing and thawing.

\section{Clinical application}

Time profiles of plasma niflumic acid-concentration after an oral administration of $740 \mathrm{mg}$ talniflumate are illustrated in Fig. 3, and the pharmacokinetic parameters are listed in Table 2. No unchanged talniflumate was found in plasma, even at $10 \mathrm{ng} / \mathrm{ml}$ of the lower limit of quantitation, and niflumic acid reached $\sim 0.2 \mu \mathrm{g} / \mathrm{ml}$ of the maximum concentration at $2.5 \mathrm{~h}$. The present results are in accordance with recent data published by Lee et al. ${ }^{4}$

\section{Conclusion}

In conclusion, our LC/MS/MS method is a very simple, sensitive, and accurate way to simultaneously determine talniflumate and its active metabolites, niflumic acid in human

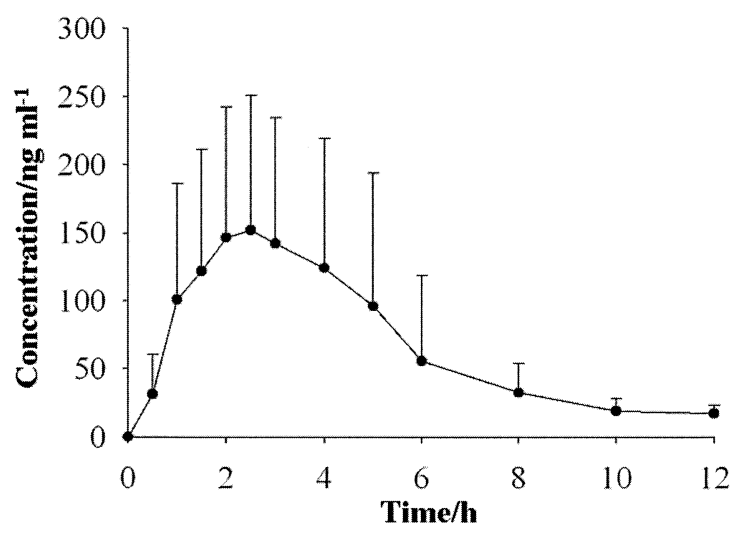

Fig. 3 Time course of the plasma concentrations of niflumic acid in healthy subjects after a single $740-\mathrm{mg}$ oral dose of talniflumate (mean \pm S.D., $n=24$ ).

plasma, and is suitable for in vitro and in vivo pharmacokinetic studies of those compounds. 
Table 2 Pharmacokinetic parameters of niflumic acid after a single oral administration of talniflumate $740 \mathrm{mg}$ in 24 healthy volunteers

\begin{tabular}{|c|c|}
\hline Parameter & Mean \pm S.D. \\
\hline$C_{\max } / \mathrm{ng} \mathrm{ml}^{-1}$ & $187.9 \pm 110.3$ \\
\hline$T_{\max } / \mathrm{h}$ & $2.5 \pm 1.0$ \\
\hline $\mathrm{AUC}_{\mathrm{t}} / \mathrm{ng} \mathrm{h} \mathrm{ml^{-1 }}$ & $795.5 \pm 467.0$ \\
\hline$t_{1 / 2} / \mathrm{h}$ & $3.0 \pm 0.9$ \\
\hline
\end{tabular}

\section{References}

1. Martindale, The Extra Pharmacopoeia, 33rd ed., 2002, Royal Pharmaceutical Society of Great Britain, 62.

2. M. Los, J. E. Boned, and C. Piccinali, Farmaco, 1981, 36,
372.

3. D.-J. Jang, J.-S. Park, H.-R. Ko, J.-P. Jee, J.-K. Kim, S.-T. Kim, and C.-K. Kim, Biomed. Chromatogr., 2005, 19, 32.

4. H.-W. Lee, K.-J. Won, S.-H. Cho, Y.-H. Ha, W.-S. Park, H.-T. Yim, M. Baek, J.-H. Rew, S.-H. Yoon, S.-V. Yim, J.-H. Chung, and K.-T. Lee, J. Chromatogr., B, 2005, 821, 215.

5. H.-J. Kim, Y.-H. Han, S.-J. Chung, M.-H. Lee, and C.-K. Shin, Arch. Pharm. Res., 1996, 19, 297.

6. C. Cuechot and P. Nicolle, J. Chromatogr., A, 1984, 303, 440.

7. G. Huin, F. Bree, and J. P. Tillement, J. Chromatogr., 1981, 223, 351

8. E. J. Park, D. H. Na, Y.-H. Shin, and K. C. Lee, J. Chromatogr., B, 2009, 876, 159.

9. W. Kang, Anal. Sci., 2006, 22, 1597.

10. W. Kang, J.-H. Jeong, E. Ma, and K.-I. Kwon, J. Pharm. Biomed. Anal., 2007, 44, 1178. 